\title{
Kernos
}

Revue internationale et pluridisciplinaire de religion grecque antique

$4 \mid 1991$

Varia

\section{Formulations possibles du serment et conceptions religieuses en Grèce ancienne}

\author{
Danièle Aubriot-Sévin
}

\section{OpenEdition \\ Journals}

\section{Édition électronique}

URL : http://journals.openedition.org/kernos/291

DOI : 10.4000/kernos.291

ISSN : 2034-7871

\section{Éditeur}

Centre international d'étude de la religion grecque antique

\section{Édition imprimée}

Date de publication : 1 janvier 1991

Pagination : 91-103

ISSN : 0776-3824

\section{Référence électronique}

Danièle Aubriot-Sévin, « Formulations possibles du serment et conceptions religieuses en Grèce ancienne », Kernos [En ligne], 4 | 1991, mis en ligne le 11 mars 2011, consulté le 01 mai 2019. URL: http://journals.openedition.org/kernos/291 ; DOI : 10.4000/kernos.291 
Kernos, 4 (1991), p. 91-103.

\section{FORMULATIONS POSSIBLES DU SERMENT ET CONCEPTIONS REIIGIEUSES EN GRÈCE ANCIENNE}

Loin de prétendre ici proposer une nouvelle étude générale concernant le serment en Grèce ancienne, nous voudrions simplement montrer que sa formulation peut s'effectuer selon deux modalités différentes, et que cela est solidaire de certaines conceptions religieuses propres à la Grèce antique.

La première de ces formulations est liée à la malédiction : un personnage désireux de garantir solennellement la véracité d'une déclaration ou la solennité d'un engagement, se voue lui-même à sa perte s'il ment. Ainsi fait Créon dans $E$ dipe-Roi, pour donner de sa loyauté envers Edipe la plus solide affirmation :

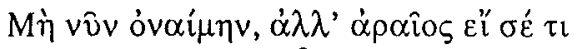

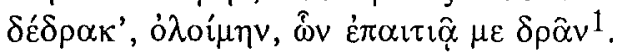

$1 \quad O . R ., 644-645$ : «Que toute chance m'abandonne et que je meure à l'instant même sous ma propre imprécation, si j'ai jamais fait contre toi rien de ce dont tu m'accuses». - Sur toutes ces questions, de plus amples précisions sont données dans la version dactylographiée de ma thèse : Recherches sur la prière dans les conceptions religieuses de la Grèce ancienne, jusqu'à la fin du Vème siècle av. J.C. (soutenue à la Sorbonne en janvier 1990), p. 757 sq. - De la bibliographie relative au serment, qui est immense, on peut extraire les titres suivants : E. ZIEBARTH, De jurejurando in jure græco quæstiones, Göttingen, 1892; L. Отт, Beiträge zur Kenntnis des griechischen Eides, Leipzig, 1896; G. GLOTZ, art. Jusjurandum, in Dict. Ant., III, 1 (1900), p. 748-775; R. HirzeL, Der Eid, Leipzig, 1902; E. ZiEBARTH, art. Eid, in RE, V, 2 (1905), col. 2076-2083; P. Stengel, Die griechischen Kultusaltertümer, Münich, 1920 (1e éd. 1898), p. 85-88; A.E. CRAWLEY, art. Oath, in E.R.E., IX (1917), p. 430-434; É. BENVENISTE, L'expression du serment dans la Grèce ancienne, in RHR, 134 (1948), p. 81-94; repris ensuite, avec des modifications de détail, dans Le vocabulaire des institutions indo-européennes, Paris, 1969, II, p. 165 sq.; J. BollaCK, Styx et serments, in REG, 71 (1958), p. 1-35; J. RUDHARDT, Notions fondamentales de la pensée religieuse et actes constitutifs du culte dans la Grèce classique, Genève, 1958, p. 202 sq.; J.F. PRIEST, "Opкıx in the Iliad and Consideration of a Recent Theory, in JNES, 23 (1964), p. 48-56; J. PLESCIA, The Oath and Perjury in Ancient Greece, Florida State Univ. Pr., 1970;

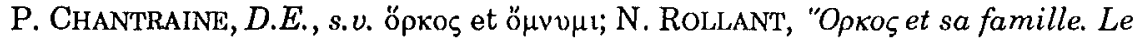
rituel de la prestation du serment dans l'Iliade et l'Odyssée d'Homère, in 
Cette protestation nous montre que jurer pouvait consister en un simple souhait, pourvu qu'il remplît certaines conditions. Elles étaient doubles : d'abord que ce souhait ne portât pas sur moins que sur la perte du jureur (c'est-à-dire fût une dóó); ensuite qu'il précisât nettement les cas pour lesquels il était valable ("si je mens», "si j'ai cette pensée»). On voit que les dieux ne sont pas invoqués, qu'ils n'ont pas le moins du monde à intervenir : le jureur est le sujet du verbe (exprimé à la première personne de l'optatif), et aucune volonté extérieure n'est sollicitée.

Il convient de remarquer corrélativement que le serment s'effectue rarement sans gestes, mais que ceux-ci, également, sont loin d'être toujours mis en rapport avec une action sacrificielle accomplie en l'honneur de dieux personnels. Certes, on trouve décrits des sacrifices juratoires ${ }^{2}$ : ils sont alors explicitement corrélatifs d'une prise à témoin des dieux. Mais on trouve aussi, et souvent, l'évocation d'un simple contact, pour lequel l'appel à l'attention des dieux est facultative. Lorsqu'au chant XIV de l'Iliade Sommeil, sollicité par Héra d'endormir Zeus, veut se ménager (en contrepartie de ce service) des assurances solides, il exige de la déesse un serment «par l'eau inviolable du Styx, en touchant d'une main le sol nourricier et, de l'autre, la mer étincelante - afin que les dieux d'en bas entourant Cronos nous servent de témoins» (v. 271-275); et l'on possède d'autres témoignages de serments prononcés manibus demissis ${ }^{3}$. Quel est le sens de ce geste? Aurait-il vraiment pour but d'attirer l'attention des dieux d'en bas ? Mais alors de qui Ménélas serait-il désireux d'éveiller l'attention quand, vexé d'avoir été lésé dans la course de chars, il requiert Antiloque de prêter un serment: "Tiens ! Antiloque, viens ici, nourrisson de Zeus, et, comme il est de règle, debout, en face de tes chevaux et de ton char, portant le souple fouet avec lequel tu menais tout à l'heure, la main sur tes chevaux, jure donc le Maître de la terre et Ébranleur du sol que tu n'as pas, par traîtrise et volontairement, gêné la marche de mon char" (Il., XXIII, 581-585). L'attelage est-il considéré comme un élément pour ainsi dire "conducteur" entre le jureur et le dieu des chevaux (mais quel serait alors le rôle du fouet ?), ou n'est-il pas plutôt regardé comme

Documents IV du Centre LAMA (Centre de recherches comparatives sur les langues de la Méditerranée ancienne), Nice, 1979, p. 214-304.

2 Un exemple célèbre s'en trouve en $I l$., III, 271-302.

3 Sur le sens du geste de l'imposition de la main, cf. HirZEL, Der Eid, p. 28 sq.; Ch. PICARD, Le geste de la prière funéraire en Grèce et en Etrurie, in RHR, 114 (1936), p. 137-157 : p. 148 sq. 
l'instrument du délit (avec le fouet), et à ce titre l'objet apte entre tous à diffuser par contamination directe une souillure redoutable en cas de parjure $^{4}$ ? D'ailleurs, une raison dissuade d'accorder créance à l'idée que le contact pourrait être effectué pour «attirer l'attention» d'une divinité - résidant sous terre, par exemple, pour reprendre le cas du serment d'Héra; c'est la considération de la pratique courante qu'est la poignée de mains, dans laquelle il est hors de question qu'une personne humaine représente ou symbolise une divinité ou sa résidence. Le théâtre de Sophocle nous offre des exemples de serments échangés à l'aide d'une poignée de mains, seule ou garantie par des paroles. Dans Edipe-Roi, le héros, avant de partir en exil, veut s'assurer que Créon prendra soin de ses filles; il lui demande sa main et un signe d'approbation :

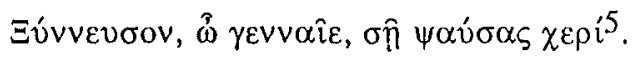

Philoctète, sentant que l'accès de son mal va en arriver au point où le sommeil le terrasse, demande à Néoptolème de ne pas l'abandonner :

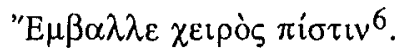

Et il considère ce geste comme un serment dans toute sa force, car il s'indigne ensuite avec véhémence de voir le fils d'Achille y manquer : "Il m'avait donné sa droite, et le voilà qui retient dans ses mains l'arc sacré d'Héraklès !» ${ }^{7}$. Enfin, dans Les Trachiniennes, quand Héraklès exige de son fils un serment complet, il commence par lui demander :

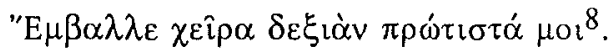

4 Sur le serment d'Antiloque, cf. L. GERNET, Droit et société en Grèce ancienne, Paris, 1955, p. 17-18; M. DetiENNE et J.P. VERNANT, Les ruses de l'intelligence. La métis des Grecs, Paris, 1974, p. 190.

5 SopH., $O . R$. 1510 : «Consens, prince généreux, touche-moi de ta main» (trad. pers.). Le thème des mains droites qui se sont serrées se retrouve à mainte

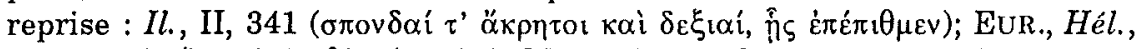

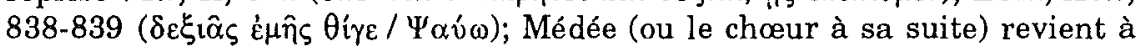
tout moment sur ce souvenir (Médée, v. 21-22; 161; 169; 208; 414; 439; 492; 496).

6 Soph., Phil., 813 : «Donne-moi ta main et ta foi tout ensemble»; (le héros

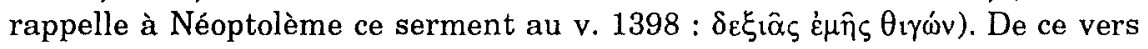
on pourrait rapprocher celui que prononce Edipe pour réclamer l'appui de

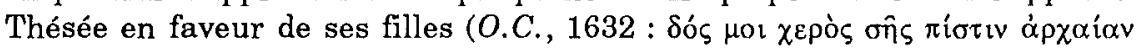

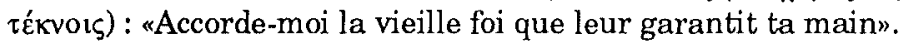

7 SopH., Phil., 942-943.

8 Soph., Trach., 1181 : «Mets d'abord ta main dans la mienne». 
On peut même aller plus loin. Indépendamment de la mention explicite des dieux ou d'une malédiction, les "droites échangées» apparaissent comme un gage qui vaut autant, voire plus qu'un serment verbalement exprimé, comme le laisse entendre la nourrice de Médée quand elle expose les malheurs de sa maîtresse délaissée par Jason :

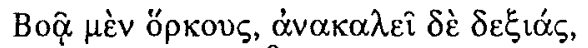

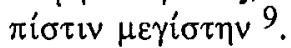

Par conséquent la poignée de mains qui engage les contractants en les mettant l'un avec l'autre en contact direct, et sans recourir à un intermédiaire sacrificiel, fait de leurs propres personnes, pour ainsi dire, l'instrument du sacrifice ou l'objet sacralisant. Les engagements réciproques - accords ou accordailles - scellés ainsi provoquaient une alliance «à la vie à la mort», comme on dit. Il faut apparemment comprendre que, comme dans la pratique de l'échange des sangs en Inde, les deux contractants rendaient sensible par cette poignée de mains le fait qu'ils ne faisaient plus qu'un. Dans ces conditions, il est bien évident qu'un acte hostile de l'un envers l'autre devenait proprement "suicidaire». Sans doute ne serait-il pas opportun de rejeter catégoriquement toute idée d'honneur des contractants. Mais s'il peut convenir d'accueillir en quelque mesure cette idée, et d'accorder quelque importance à la notion morale de "bonne foi», c'est en se souvenant qu'a déjà été aperçue une certaine subordination du code de l'honneur réputé seulement humain (accorder à chacun la $\tau \imath \mu \eta ́$ qui lui revient) par rapport aux droits que la religion impose de ne pas léser, et qui peuvent se résumer sous le nom de $\Delta i \kappa^{10}{ }^{10}$. En ces types

9 Eur., Médée, 21-22 : «À grands cris (elle) invoque les serments, les mains échangées, gage suprême».

10 Sur l'ancienneté «indubitable» du culte de Fides en Italie, et sur les rapports

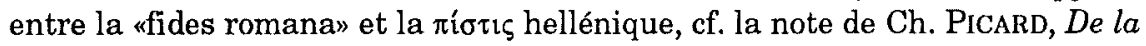

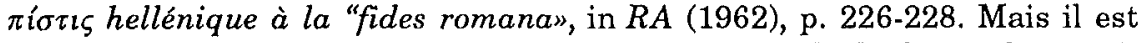
difficile de déterminer à partir de quand la notion morale de «bonne foi» a pris le pas sur l'obligation religieuse de tout faire pour se soustraire à une souillure (en tout cas, on ne peut affirmer sans restriction, comme faisait J. GIRARD, Le sentiment religieux en Grèce, d'Homère à Eschyle, Paris, 1879, p. 95, que la Justice «est un sentiment, une force morale»; dans un sens opposé, voir W. BURKERT, Homo Necans, Berlin, 1972 [R.G.V.V., 32], tr. angl., Homo Necans. The Anthropology of Ancient Greek sacrificial Ritual and Myth, Berkeley/Los Angeles/London, 1983, p. 37, n. 11). Sur cette notion, on pourra consulter (outre

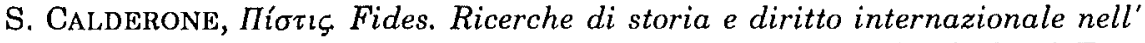
antichita, Messine, 1964, p. 38; 45 sq.) H. RoISMAN, Loyalty in Early Greek Epic and Tragedy, Königstein, 1984 (Beitr. zur klass. Philologie, 155). 
d'engagement en tout cas, un appel à la garantie divine est loin d'apparaître au premier plan - à supposer même qu'il soit opportun d'en faire mention. Les serments formulés à l'aide d'une simple malédiction contre le contrevenant éventuel sont fondés sur une croyance à l'efficacité propre des gestes et des mots. Précisons bien qu'il serait inadéquat, en ces contextes, de parler de magie : cette croyance en une efficacité automatique en effet, se fonde sur une foi - qui est d'ordre religieux - en la toute-puissance de $\Delta i \kappa \eta$. Mais on ne saurait non plus, au rebours, parler de code de l'honneur : ce qui rend un serment absolument contraignant, ce n'est pas l'obligation morale de ne pas manquer à la parole donnée, c'est en quelque manière l'instinct de conservation. Car sous les notions qui devaient prendre à l'époque classique (et garder jusqu'à nous) une valeur morale - comme la nécessité d'éprouver un sentiment de respect envers les parents, les tombeaux, les sanctuaires, ou les serments - il convient de restituer l'obligation de maintenir dans leur intégrité des relations où chacun se tient strictement et attentivement à sa place, au rang que $\Delta$ íкn lui assigne; faute de quoi on sent percer partout la crainte de se voir atteint automatiquement, en retour d'un manquement de nature à compromettre l'harmonieux développement de la fécondité, par une lésion identique.

En somme, ce qui est supposé par ce genre de pratiques (comme le serment fondé sur la malédiction conditionnelle et appuyé de contacts lourds de contaminations virtuelles), c'est une croyance en une religion de la puissance, au sein de laquelle les forces de contagion agissent d'elles-mêmes (contagion bénéfique pour un jureur fidèle, maléfique pour un parjure), sans que les dieux soient appelés à jouer aucun rôle dans ce processus, censé se dérouler automatiquement.

Une autre possibilité de formulation consistait à invoquer les dieux comme témoins, avec plus ou moins de solennité : un simple vì tòv $\Delta i ́ \alpha$ pouvait ou bien n'être guère plus que ce que nous appelons encore un «juron» ${ }^{11}$, ou bien constituer une attestation d'une gravité indubitable.

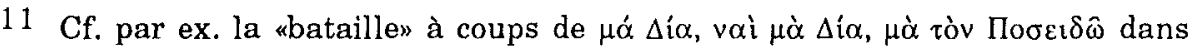
Aristoph., Cav., 336-338. Nombreux ex. dans R. Kuhnlein, De vi et usu precandi et jurandi formularum apud decem oratores atticos, Neustadt, 1882, p. 3 sq.; cf. ZIEBARTH (1892), p. 7; J. RUDHARDT, Notions fondamentales, p. 205; Plescia, p. 4. P.E. PFIFFner, Die Götteranrufungen in den Werken der drei Tragiker in den Komödien des Aristophanes und in den Dialogen Platons und Xenophons, Einsiedeln, 1931, rassemble commodément, ici et là (p. 7 sq.; 23-24; $35 ; 45 ; 50 ; 58-59$ ), un bon nombre de textes utilisables comme exemples; mais il 
Ainsi, le premier serment que prête Achille au chant I de l'Iliade, quand il jure à Calchas de le défendre, représente une promesse tout à fait solennelle :

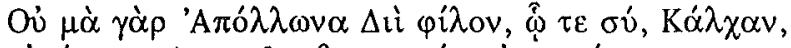

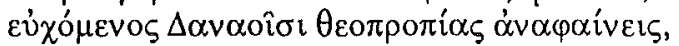

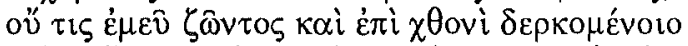

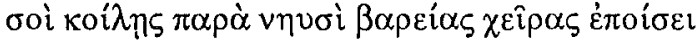

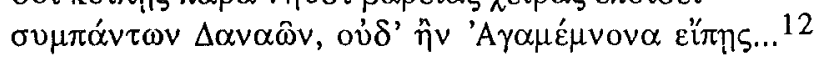

Non seulement un dieu est attesté, mais la relative nous prouve que le nom de ce dieu est invoqué en rapport avec les fonctions religieuses publiques du devin, que donc nous sommes aussi loin que possible d'une attestation machinale, (quoique par ailleurs Achille ne tienne aucun "objet sacralisant" en main). On constate que ce type de serment comporte simplement, après la prise à témoin du dieu, l'énoncé d'un engagement pris : ici, celui de protéger Calchas; on pourrait citer encore l'autre engagement bien imprudent qu'assume Hector (sceptre en main, cette fois), de donner à Dolon les chevaux d'Achille ( $I l$., X, 329331) : "Zeus le sache, Zeus, l'époux retentissant d'Héré ! aucun autre parmi les Troyens ne montera sur ce char. Seul, je te l'assure, tu te pareras de cette gloire à tout jamais». En ce genre de démarche, l'appel au témoignage d'une personne divine nommément désignée est ce qui constitue la garantie du serment.

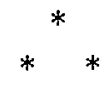

Nous nous trouvons donc pour le moment devant deux types d'initiatives qui ont l'air parfaitement distinctes : l'une consiste en un souhait, formulé comme il est naturel à l'optatif, sans rapport nécessaire avec une divinité, mais constituant une imprécation contre soimême en cas de dol; l'autre consiste à prononcer par devant une divinité une promesse solennelle (qui peut porter sur le passé, sur le présent, ou sur l'avenir) exprimée à l'indicatif. Ces deux types de

faut prendre garde qu'il mélange serments, invocations, et adjurations (par ex.

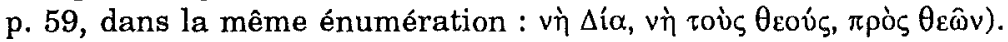

$12 I l$, I, 86-90 : «Non, par Apollon cher à Zeus, à qui, Calchas, va ta prière, lorsque tu veux aux Danaens révéler les arrêts du ciel, non, tant que je vivrai, tant qu'ici-bas j'aurai les yeux ouverts, nul, près de nos nefs creuses, ne portera sur toi sa lourde main, nul entre tous les Danaens, quand tu nommerais même ici Agamemnon..." 
démarches, caractérisés par des expressions et des modalités bien distinctes, apparaissent fondés sur des conceptions religieuses qui nous semblent, à nous modernes, radicalement différentes, en ce que les unes privilégient la religion de la puissance, tandis que dans les autres, le recours aux personnes divines est essentiel.

Mais la religion grecque ignore ces distinctions bien tranchées et en l'occurrence, ces deux modes de formulation du serment sont loin de s'exclure réciproquement. Ainsi, quand Agamemnon, au chant XIX de l'Iliade ${ }^{13}$, jure à Achille qu'il a respecté Briséis, il commence par prendre les dieux à témoin; il affirme ensuite qu'il ne l'a pas touchée; puis il appelle sur soi, pour le cas où il aurait menti, les maux qui accablent les parjures : on a donc une addition des deux procédés ${ }^{14}$.

13 Il., XIX, 258-265 : "Que Zeus d'abord m'en soit témoin, le plus haut, le plus grand des dieux ! et la Terre et le Soleil ! et les Érinyes qui, sous terre, châtient les morts parjures à un serment! non, jamais je n'ai porté la main sur la jeune Briséis, ni par désir avoué de son lit, ni pour nulle autre cause. Elle est restée intacte, toujours, dans ma baraque. Et, si je commets ici le moindre parjure, que les dieux me fassent souffrir les mille maux qu'ils font souffrir à qui les a offensés en jurant!»

14 Il est frappant cependant qu'il rattache même cette malédiction conditionnelle, pourtant exprimée comme il se doit à l'optatif, aux dieux : Ei $\delta \varepsilon \dot{\varepsilon} \tau \imath \hat{\omega} v \delta$ '

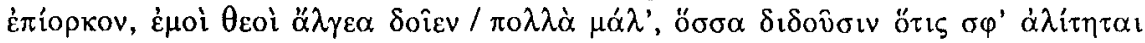

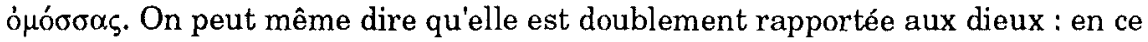
qu'ils sont censés être les distributeurs des maux d'une part; en ce que le parjure les offense personnellement de l'autre - tant il est vrai que religion de la personne et religion de la puissance ne sont pas dissociables; cf. J. RUDHARDT, Notions fondamentales, p. 204 : «L'invocation ne figure pas nécessairement au début du serment. Elle crée pourtant un lien entre l'homme et les dieux, une solidarité telle que nous verrons ceux-ci lésés par le parjure». Cependant, comme J. RudhaRdT le précisait lui-même un peu plus haut (ibid.): "L'invocation dans le serment n'est pas exactement un appel : les noms des dieux n'y figurent pas au vocatif. Elle comprend les formules vì roì $\theta$ coú

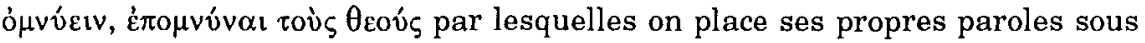
l'autorité et sous la garantie des dieux". - Cette qualité de témoins, ou de

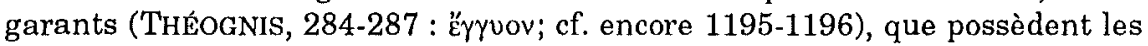
dieux, a déjà été soulignée à mainte reprise : cf. par ex. GERNET \& BOULANGER, Le Génie grec dans la religion, Paris, 1932, p. 221-222; L. GeRneT, Anthropologie de la Grèce antique, Paris, 1968, p. 215, ou Benveniste, Vocabulaire, II, p. 173-174, à propos de l'impératif ‘̆ $\sigma \tau \omega$ et de la qualité irrécusable du témoignage oculaire. De fait assez souvent, au lieu de comporter à la fois attestation et imprécation, le serment ne comporte que l'un des deux : on peut jurer sans faire d'imprécation (SoPH., Ant., 304-305; 758; El., 626-627; $881 ; 1063-1065 ; 1239$; Phil., 1289 , par ex.), mais aussi sans citer les dieux (O.R., 644-645). Quoique l'appel aux dieux soit facultatif, leur garantie est considérée comme acquise : "De là vient... cette idée qu'on offense les dieux donnés 
D'un autre côté, dans le premier chant de l'Iliade, Achille est amené à prononcer un nouveau serment après le premier, déjà mentionné; c'est alors non plus une addition, mais une contamination que l'on trouve :

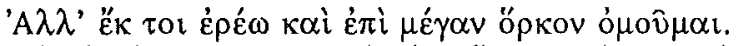

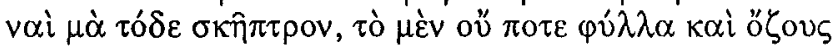

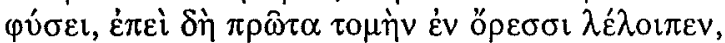

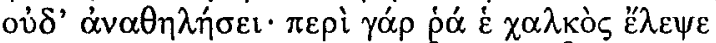

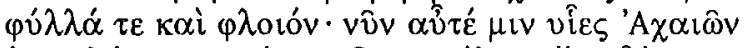

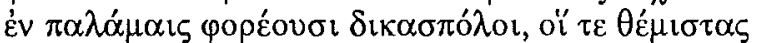

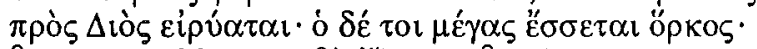

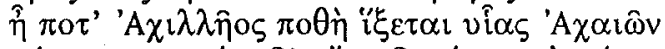

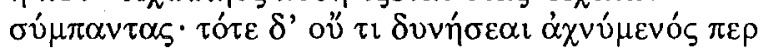

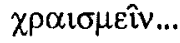

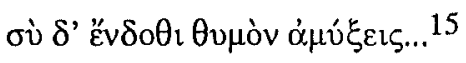

comme caution, non seulement quand on prête un faux serment, mais encore quand on refuse créance au serment d'autrui" (G. GLOTZ, art. jusjurandum, p. 748); cette idée ne perd rien de sa force s'il s'agit d'un serment prononcé sous forme de la seule imprécation, c'est-à-dire sans appel explicite au témoignage des dieux. Ainsi dans O.R., Edipe se voit prié de respecter la parole de Créon qui vient de se vouer à la mort en cas de mensonge : "Respecte sa parole, les dieux en sont garants", lui dit Jocaste (v. 647); or Créon n'avait pas attesté les dieux (v. 644-645).

15 Il. I, 233-244 : «Eh bien ! je te le déclare et j'en jure un grand serment. - Ce bâton m'en soit témoin, qui jamais plus ne poussera ni de feuilles ni de rameaux, et, maintenant qu'il a quitté l'arbre où il fut coupé dans la montagne, jamais plus ne refleurira ! Le bronze en a rasé le feuillage et l'écorce, et le voici maintenant entre les mains des fils des Achéens qui rendent la justice et, au nom de Zeus, maintiennent le droit. Ce sera là pour toi le plus sûr des serments. - Un jour viendra où tous les fils des Achéens sentiront en eux le regret d'Achille; de ce moment-là, malgré ton déplaisir, tu ne pourras plus leur être en rien utile... Alors, au fond de toi, tu te déchireras le cœur...» Notons qu'il n'est pas tout à fait juste d'affirmer qu'Achille dise à Agamemnon (Il., I, 234 sq.) que le sceptre lui

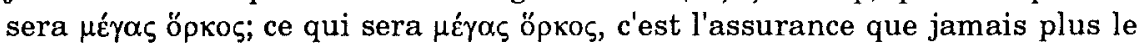
feuillage ne reverdira sur cette branche morte; il faut comprendre que parallèlement Achille engage sa tête (aussi vrai que le sceptre est privé de sève) sur la promesse qu'Agamemnon se repentira de lui avoir infligé cet affront. J. BolLACK a bien vu (p.15) que ce «bâton de juridiction» était particulièrement en situation quand il s'agissait d'Agamemnon; surtout, R. Hiersche, dans la "Note additionnelle relative à l'étymologie d'ópко et d'ó $\mu v v ́ v \alpha$ " qui suit l'art. de J. Bollack (ibid., p. 35 sq.), dégage très clairement le fait que le öpкos n'est pas positivement tò $\sigma \kappa \hat{n} \pi \tau$ pov. - Que le serment utilise dans une large mesure le rôle de l'action, du geste, a été souligné avec insistance par K. MAROT, Der Eid als Tat, Szeged, 1924. Sur les vertus conjuguées du sceptre, du geste, autant que de la parole, cf. L. GERNET, Anthropologie, p. 216, n. 172. 
Il est visible que ce serment est construit selon le modèle des promesses faites par devant les dieux; et cependant, ce qui suit vai $\mu \grave{\alpha}$ n'est pas le nom d'un dieu, mais celui d'un "objet sacralisant", comme dit Benveniste : c'est le sceptre, longuement décrit, des Achéens. Il nous semble qu'on fait fausse route en prétendant qu'Achille jure par ce sceptre parce qu'il l'érige au rang de dieu ${ }^{16}$ - aussi bien le jette-t-il à terre après qu'il a fini de parler ${ }^{17}$. Bien plus probablement, selon nous, a-t-on affaire à une véritable superposition d'un mode de serment, à fonctionnement automatique, et d'un autre, qui commet à la divinité le châtiment du parjure. Il ne semble pas douteux que, si Achille insiste comme il le fait sur la stérilité désormais définitive du bois autrefois vivant qui a servi à faire le sceptre, c'est pour indiquer l'idée : «Puisséje être soumis à la même sécheresse impuissante, si les choses ne se passent comme je l'annonce». Mais les présupposés religieux d'Homère font qu'il serait malséant pour le héros d'appeler ainsi sur sa tête une imprécation dont l'action serait aussi nettement spontanée et indépendante de la volonté divine. Il faut s'aviser que même le toutvenant de l'armée achéenne ne pense pas devoir supprimer une solennelle invocation à Zeus en tête d'un souhait qui pourtant (la syntaxe en fait foi) ne le concerne pas directement :

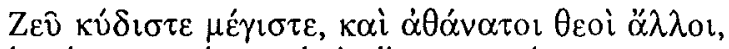

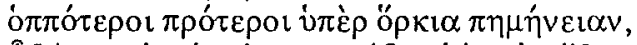

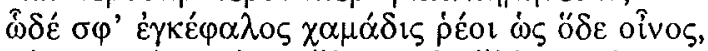

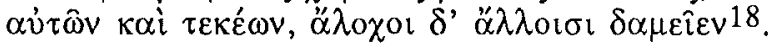

16 Comme l'a supposé S. Benardete, Achilles and the Iliad, in Hermes, 91 (1963), p. 1-16: p. 15; ou plutôt, l'auteur prétend que les dieux pour Achille ne sont rien de plus que son sceptre, i.e. son pouvoir (ce n'est, soit dit en passant, pas le sien), qui perd toute sa force quand il le jette; et il ajoute que n'importe quelle branche aurait aussi bien fait l'affaire. Cela nous semble relever du contresens. $\Pi$ n'en reste pas moins que, comme le dit (après DeUBNER) L. GERNET, Anthropologie, p. 205, «le sceptre n'est pas seulement le signe, mais le siège d'une force religieuse".

17 Il., I, 245; cf. J. BOLLACK, art. cit., p. 15.

18 Il., III, 298-301 : «O Zeus très glorieux, très grand! et vous tous, dieux immortels! quel que soit celui des deux peuples qui le premier viole ce pacte, tout comme je répands ce vin, que soit répandue à terre la cervelle de tous les siens, pères et enfants, tandis que leurs femmes subiront un maître étranger». Quelques vers plus loin, un quatrain analogue est attribué encore aux deux armées, mais cette fois, il n'y a plus d'ópó à l'optatif; elle est remplacée par une demande à Zeus (Il., III, 320-324) : «Zeus Père, maître de l'Ida, très glorieux, très grand! fais que celui des deux qui à nos peuples apporta ces soucis (tòv dòs 
Il nous paraît donc qu'Achille élimine volontairement une prise à témoin des dieux dans une affaire pour laquelle il ne leur demande pas leur avis : le sceptre sur lequel il jure, en même temps symbolise la vie sociale et pour ainsi dire juridique des Achéens ${ }^{19}$, bafouée par l'égoïsme d'Agamemnon, et suggère l'idée que le héros s'engage tout entier dans cette promesse, sans passer par aucun intermédiaire (tant il est sûr de sa décision inflexible); mais il nous paraît aussi que les bienséances épiques l'empêchent d'utiliser la formulation et le mode convenant pour un déclenchement automatique ${ }^{20}$ : ce serment constitue en quelque sorte un compromis entre la formulation qui traditionnellement sert à prendre les dieux à témoin, et la formulation qui se passe de l'attestation des dieux. On pourrait dire qu'il équivaut à une manière d'aposiopèse.

Ces quelques exemples suffisent, espérons-nous, à la fois pour établir la distinction entre les deux formulations "pures" possibles (celle qui recourt à l'ópó automatique et celle qui commet explicitement la vengeance aux dieux), et pour montrer que des déformations stylistiques peuvent brouiller les faits. Mais des intentions artistiques ne sont pas seules en cause; et ce que nous avons appelé l'addition des

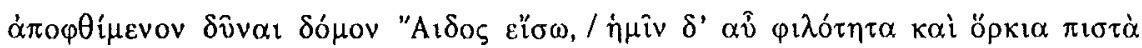
$\gamma \varepsilon v \varepsilon \delta \sigma \theta \alpha$ ) meure et entre chez Hadès, tandis que nous, nous conclurons un pacte loyal de bonne amitié». Ces prières ont retenu l'attention de F. ScHWENN, Gebet und Opfer. Studien zum Griechischen Kultus, Heidelberg, 1927, p. 30-32.

19 Sur le sceptre, symbole du pouvoir sacré, surtout lié à l'aspect juridique de la première fonction, cf. A. YosHIDA, La structure de l'illustration du bouclier $d^{\prime}$ Achille, in $R B P h, 42(1964)$, p. $5-15:$ p. 14, n. 9, avec la référence à E. Janssens, Le pouvoir et le sacré, Bruxelles, I, 1962 (Annales du Centre d'Etudes des Religions), p. 91.

20 Il est remarquable en effet que l'épopée ne fasse, sauf exception, qu'un usage discret, voire pour ainsi dire déguisé (comme ici) de ce genre de serment comportant un appel de malédiction conditionnelle sur sa propre tête. Ce n'est pas qu'elle l'ignore (cf. par ex. Il., XIX, 264-265 et surtout III, 300-301), mais elle en use avec ménagement, et en propose parfois des atténuations : «Que je ne sois plus appelé le père de Télémaque", dit Ulysse à Thersite (Il., II, 260) en lui promettant un châtiment exemplaire s'il recommence; en revanche, on trouve plus souvent l'autre forme d'engagement par serment, qui consiste à prononcer par devant une ou des divinités une promesse solennelle (qui peut porter sur le passé ou sur l'avenir) exprimée à l'indicatif, ou à rendre ces divinités témoins de la véracité de l'affirmation. 
deux procédés a connu une fortune dont les poèmes homériques, qui en usent parcimonieusement, n'auraient pas permis d'imaginer l'étendue si nous avions dû nous fonder sur leur seul témoignage. Une grande partie des textes épigraphiques portant des serments entre États est là pour nous assurer qu'à l'invocation solennelle des dieux en début d'engagement, s'ajoutait à la fin, après que les clauses avaient été

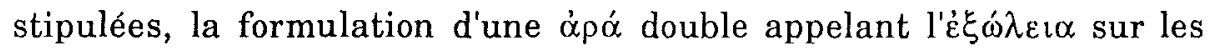
parjures, et la prospérité sur ceux qui seraient fidèles à leur serment ${ }^{21}$. En sorte que ces formulations qui apparemment procèdent de conceptions religieuses différentes (en mettant l'accent, tantôt sur la "puissance", avec son cortège de processus automatiques, tantôt sur l'appel aux personnes divines), loin de s'exclure comme nous aurions eu tendance à le présumer, de notre point de vue cartésien de modernes, apparaissent au contraire complémentaires.

Nous ne serons pas surpris de voir que les textes littéraires de l'époque classique, au gauchissement près qu'exige le propos de chaque auteur, nous offrent des exemples parallèles. C'est ainsi que par delà les siècles et la coloration morale différente des œuvres, un autre témoignage de cette dualité possible des formes du serment se trouve dans Les Trachiniennes de Sophocle. À cette différence près que tout, dans la bouche d'Héraklès, est volontairement rapporté à Zeus son père, on distingue encore très bien, dans les parties successives de l'engagement auquel il soumet Hyllos, les deux étapes que nous avons cru pouvoir distinguer ailleurs. Le héros commence par ordonner à son fils de jurer par Zeus :

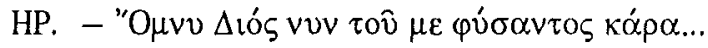

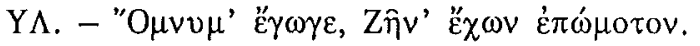

Il lui demande ensuite de se vouer à sa perte en cas de trahison :

21 Cf. par ex. le fameux serment de Théra (cf. P.M. SchuhL, Essai sur la formation de la pensée grecque, Paris, 1949 [1e éd. 1934], p. 60) gravé sur une inscription de Cyrène; le serment d'alliance entre Athènes et Corcyre (datant de 375-374) = I.G., II ${ }^{2}, 97\left(S y l l .{ }^{3}, 151\right)$, cité et commenté par J. Poullloux, Choix d'inscriptions grecques, I, Paris, 1960, p. 105 sq. (et en partic. p. 107 pour le

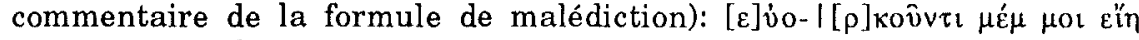

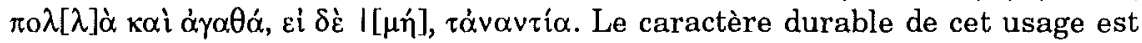
bien souligné par PFAFF dans son art. Exsecratio, in RE, Suppl. IV (1924), col.

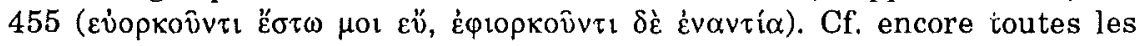
références mentionnées par W. SPEYER, (art. Fluch, in R.A.C., VII [1969], col. 1160-1288), col. 1199-1200 et 1205-1206. 


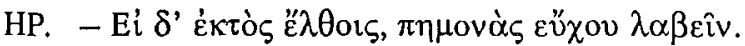
Y $\Lambda .-\ldots$
$\varepsilon v ้ \chi 0 \mu \alpha \iota^{22}$.

Il convient peut-être de chercher dans cette dualité d'expression l'explication d'une formule de l'épopée dont les deux termes semblent autrement curieusement redondants; il s'agit de la tournure ö $\mu \circ \sigma \varepsilon ́ v \tau \varepsilon$

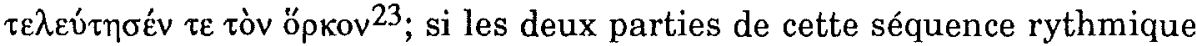
possèdent bien chacune leur unité sémantique, ainsi qu'il est logique de le supposer, il faut saisir une nuance entre l'un et l'autre terme. Or il nous semble justement que nous avons rencontré une explication possible, conforme aux usages du serment : ö $\mu \circ \sigma \varepsilon ́ v \tau \varepsilon$ renverrait à

22 SoPH., Trach., 1185-1189:

«Hér. - Jure par la tête de mon père, Zeus...

Hy. - Je le jure, et j'en prends Zeus même pour garant.

Hér. - Et si tu manques à ta parole, voue-toi toi-même au malheur.

Hy. - ... J'en fais le vœu».

(Il est évident qu'on peut formuler des réserves sur la traduction, qui présente

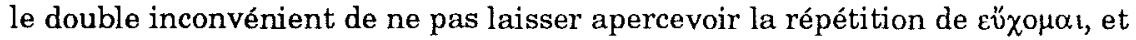
d'estomper ainsi le choix théologique qui est fait de rapporter l'effet de la malédiction à la volonté divine). - On trouve la même séquence des deux volets d'un serment quand Médée veut s'assurer de l'aide d'Egée (Médée, 746 sq.):

M. «- Jure (ö $\mu v v)$ par le sol de la Terre, par le Soleil, père de mon père, et par toute la race des dieux réunis...

É. - De faire ou de ne pas faire quoi ? Parle.

M. -De ne jamais, toi-même, me chasser de ton territoire, et si un de mes ennemis veut m'en arracher, de ne pas me livrer à lui, toi vivant, de ton plein gré.

É. - J'en jure par la Terre (ö $\mu v v \mu \mathrm{\iota} \Gamma \alpha \hat{\imath} \alpha v \kappa \tau \lambda . .$.$) , par la brillante lumière du$ Soleil et par tous les dieux : j'observerai ce que tu dis.

M. - I suffit. Mais si tu n'observes pas ce serment, quel châtiment te souhaiter

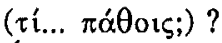

É. - Celui qui frappe les mortels sacrilèges».

Il est donc bien net qu'il y a d'une part attestation et engagement, et de l'autre sanction par la malédiction.

23 Cf. Il., XIV, 280; Od., II, 378; X, 346; et, au pluriel : XII, 304; XV, 438; XVIII, 59. Notre interprétation de cette formule va à l'encontre de ce qui a été avancé par J. BollaCK qui (p. 7-8) suggère que öpкov $\tau \varepsilon \lambda \varepsilon v \tau \hat{\alpha} v$ signifie "prononcer les noms rituels". Il nous semble au contraire que ó $\mu v \hat{v} v \alpha_{l}$ s'entend pour l'attestation et l'engagement (comme l'indiquent les passages de la note précédente tout autant qu'Il., XXI, 373; XXIII, 584-585, par ex.), et que öprov $\tau \varepsilon \lambda \varepsilon v \tau \hat{\alpha} v$ renvoie à la sanction exprimée par la malédiction; quant à l'expression öpкov ó $\mu v v ́ v \alpha$, elle pourrait selon nous désigner l'ensemble de la prestation. 
l'invocation des dieux, et à l'engagement proprement dit, tandis que

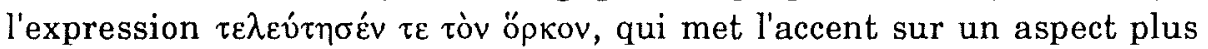
concret du serment, relatif à l'achèvement dans les faits, désignerait la partie consistant en l'ópó. Quoi qu'il en soit de cette proposition, il reste que les deux formes d'engagement coexistent le plus souvent dès l'instant qu'on a affaire à un serment quelque peu solennel. Cependant leur addition n'est pas indispensable, et l'on peut très bien s'en tenir seulement à l'une ou à l'autre; à l'extrême, d'un côté, on aboutit au juron, et de l'autre, ne demeure que la malédiction conditionnelle, plus ou moins allusive. Mais dans les deux hypothèses, la formule ne cesse pas d'être chargée d'un poids religieux que nul ne songe à mettre en doute - tant il est vrai qu'il n'y a pas, en Grèce ancienne, d'antinomie entre la religion de la puissance et celle de la personne.

Université de Picardie

Danièle AUBRIOT

Faculté de Lettres, Campus

F - 80025 - AMIENS CEDEX 